\title{
Epidémiologie de la fièvre Hémorragique de Crimée-Congo (FHCC) chez les bovins dans le département de Boboye au Niger
}

\author{
Alima MAÏNA ${ }^{1}$, Abdoulkarim ISSA IBRAHIM $^{2 *}$, Abdou ALASSANE $^{1}$ et \\ Hassane ADAKAL ${ }^{3}$
}

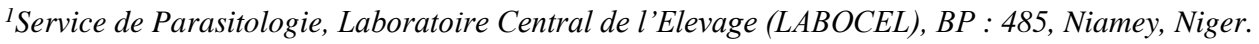
${ }^{2}$ Faculté des Sciences Agronomiques, Université Boubacar BA de Tillabéri, BP : 175 Tillabéri, Niger. ${ }^{3}$ Faculté d'Agronomie, Université Dan Dicko Dankoulodo de Maradi, BP 465 Maradi, Niger. *Auteur correspondant, E-mail: karimlebelge@yahoo.fr; Tél : (00227) 96573021.
\end{abstract}

\section{RÉSUMÉ}

La distribution et la dynamique des populations des tiques est un élément clé dans la connaissance des maladies transmises par ces vecteurs. C'est ainsi que cette étude a été conduite afin de mieux connaître l'épidémiologie de la Fièvre Hémorragique de Crimée-Congo (FHCC) dans les 8 communes du département de Boboye au Niger, où 355 sérums de bovins ont été collectés. En plus des sérums, des tiques ont été collectées sur 144 bovins, soit 18 par commune. Les sérums ont été soumis à un test ELISA (Enzyme Linked Immunosorbent Assay) indirect pour la détection d'anticorps anti-FHCC. Soixante-douze (72) éleveurs ont été interviewés sur leur connaissance de l'écologie des tiques, vecteurs du virus de la FHCC. Les résultats de l'enquête ont révélé que les éleveurs n'ont pas recours aux acaricides et que, dans leur majorité (55/72 soit $76,4 \%$ ), ils pratiquent la transhumance. L'étude a permis l'identification de 1342 tiques réparties en trois genres : Hyalomma (91,7\%), Amblyomma (5,7\%) et Rhipicephalus (Boophilus) (2,6\%). La séroprévalence globale a été de $9,1 \pm 0,03 \%$. Les communes de Harikanassou et Kiota ont été celles où les fortes prévalences ont été observées de $26,7 \pm 12,9 \%$ et $22,5 \pm 12,9 \%$. Le virus de la FHCC est en circulation chez la population animale, alors des investigations doivent être faites chez la population humaine.

(C) 2020 International Formulae Group. All rights reserved.

Mots clés : Anticorps anti-FHCC, Enzyme Linked Immunosorbent Assay Indirecte, Prévalence, Sérums, Tiques.

\section{Crimean-Congo Hemorrhagic Fever (CCHF) 's Epidemiology in cattle in Boboye's department of Niger Republic}

\begin{abstract}
To understand disease transmission by ticks, knowledge of population dynamics and distribution of these vectors are essentials. To sought that, the epidemiology of Crimean-Congo Hemorrhagic Fever (CCHF) in Niger Republic was studied by sampling 355 bovines (sera and ticks) in eight (8) local governments in Boboye's department. Eighteen (18) bovines were sampled for ticks collection per local government making them a total of 144 bovine. Indirect ELISA test (enzyme-linked immunosorbent assay) was used to detect anti- CCHF
\end{abstract}


antibodies. Seventy-two (72) farmers were surveyed on their knowledge on ticks' ecology, main vectors of CCHF virus. The results revealed that farmers are not using acaricides, and their majority (55/72 thus $76.4 \%)$ practice Transhumance. The study allowed the identification of 1342 ticks distributed in 3 genus: Hyalomma (91.7\%), Amblyomma (5.7\%) and Rhipicephalus (Boophilus) (2.6\%). The global seroprevalence against CCHF was (9.1 $\pm 0.03) \%$. Harikanassou and Kiota were the most affected local governments with respectively $(26.7 \pm 12.9) \%$ and $(22.5 \pm 12,9) \%$ prevalence. $\mathrm{CCHV}$ virus is circulating in animal population, so investigations must be made in human population.

(C) 2020 International Formulae Group. All rights reserved.

Keywords: Anti-CCHF antibodies, Indirect Enzyme Linked Immunosorbent Assay, Prevalence, Sera, Ticks.

\section{INTRODUCTION}

La fièvre hémorragique de CriméeCongo (FHCC) est une zoonose virale majeure souvent mortelle chez l'homme. Le virus de la FHCC appartient à la famille des Nairoviridae genre Orthonairovirus. C'est un arbovirus à ARN enveloppé, sphérique, de 90 à $100 \mathrm{~nm}$. Selon Zientara (2013), le virus peut être transmis par « de nombreuses espèces de tiques appartenant à différents genres, mais les vecteurs les plus efficaces et les plus courants appartiennent au genre Hyalomma. Des tiques des genres Rhipicephalus et Dermacentor sont également capables de transmettre la maladie ».

Des signes cliniques sont observés suite à l'infection chez les humains alors que chez les animaux elle est asymptomatique. Néanmoins les animaux infectés présentent une virémie pouvant durer 2 à 15 jours (Papa et al., 2017). Chez l'homme, les symptômes sont une fièvre intermittente pouvant durer 9 à 14 jours, mais également des frissons, une sudation abondante, des maux de tête importants, des courbatures, le tout parfois accompagnés de vomissements et de nausées. Dans environ $50 \%$ des cas, les malades présentent une pneumonie radiologique avec quelques manifestations cliniques (toux légères). L'infection provoque également des hémorragies digestives extrêmement sévères, avec un pronostic très défavorable.

L'une des caractéristiques les plus redoutables de ce virus est son aptitude à provoquer des épidémies nosocomiales (Saluzzo et al., 2004). Les personnes présentant le risque le plus élevé de contracter l'infection par le virus de FHCC sont les fermiers vivant dans les régions endémiques, le personnel médical, les vétérinaires et le personnel travaillant dans les abattoirs. Le taux de létalité de la FHCC s'établit à 30\% environ, la mort survenant au cours de la deuxième semaine de la maladie (OMS, 2013). Elle est endémique en Afrique, dans les Balkans, au Moyen-Orient et en Asie, dans les pays en-deçà du 50 ème degré de latitude Nord qui constituent la limite géographique du genre Hyalomma, vecteur principal de la maladie (OMS, 2013).

$\mathrm{Au}$ Niger, la première étude sur la FHCC a été faite sur les petits ruminants en 1991. Ainsi, sur 1423 sérums analysés, aucun cas positif à la FHCC n'a été obtenu. Depuis, aucune étude n'a été entrepris sur cette maladie au Niger.

L'objectif de la présente étude était de contribuer à une meilleure connaissance de l'épidémiologie de la Fièvre Hémorragique de Crimée-Congo chez les bovins dans le département de Boboye, au sud du Niger. De façon plus spécifique, il s'agissait (i) d'évaluer les perceptions qu'ont les éleveurs de l'infestation par les tiques; (ii) d'identifier les différentes espèces de tiques qui parasitent le bétail, et enfin (iii) de déterminer la séroprévalence de la FHCC chez les bovins.

\section{MATÉRIEL ET MÉTHODES}

\section{Zone de l'étude}

L'étude a été menée au cours du mois de septembre 2016 dans le département de Boboye situé dans la Région de Dosso au Niger 
(Figure 1). Le département de Boboye couvre une superficie de $4797 \mathrm{~km}^{2}$ et est subdivisé en 8 communes: Birni Gaouré, Fabirdji, Kankandi, Harikanassou, Koygolo, Ngonga, Fakara et Kiota. La population est estimée à environ 250.000 habitants (INS, 2014) et le cheptel bovin à environ 170.000 têtes. Le climat est de type sahélien. La pluviométrie annuelle (en $\mathrm{mm}$ ), relevée à Birni Gaouré a été de 575, 467, 670, 680 et 639 respectivement en 2010, 2011, 2012, 2013 et 2014 (INS, 2014).

\section{Echantillonnage et prélèvements}

Tous les bovins âgés de plus de quatre mois, sans distinction de race ni de sexe, ont été inclus dans l'étude. La base de sondage était constituée de l'ensemble du cheptel du département. Avec l'hypothèse d'une prévalence de $8,9 \%$ (selon une étude réalisée sur les bovins en Mauritanie par Saluzzo et al.(1986), le logiciel Win-Episcope (version 2.0) a été utilisé pour calculer la taille minimale de l'échantillon, qui était de 347 bovins (avec une erreur acceptée de $3 \%$ et un niveau de confiance de 95\%). Finalement, 355 bovins provenant de 9 troupeauxont été inclus dans l'étude, soit 45 bovins par commune sauf à Kiota (40).

D'autre part, un échantillonnage aléatoire simple a été réalisé pour la collecte des tiques. Ainsi, dans chacun des 9 troupeaux, les tiques ont été collectées sur deux bovins choisis au hasard, soit un total 18 bovins. Elles ont été conservées dans des pots de prélèvement contenant de l'éthanol à $70^{\circ}$ puis acheminées au LABOCEL où elles ont été identifiées selon la description des différents genres publiée par Walker et al. (2003).

Les prélèvements de sang ont été faits en même temps que les tiques par ponction de la veine jugulaire, sur tube sec. Après centrifugation, le sérum a été réfrigéré à $4{ }^{\circ} \mathrm{C}$, puis acheminé au Centre de Recherche Médicale et Sanitaire de Niamey (CERMES).

Enfin, une enquête de perception a été réalisée au moment des prélèvements grâce à des questionnaires conçus avec le logiciel sphinx (V5). Les questions posées aux éleveurs concernaient la mobilité des troupeaux (transhumance), la période d'abondance des tiques, les traitements pratiqués.

\section{Analyse sérologique des prélèvements}

L'Elisa indirect pour la recherche des anticorps anti-FHCC, témoins de l'infection des bovins, a été choisie comme test sérologique. Pour cela, le kit «CRIMEANCONGO IgG test ELISA » a été utilisé conformément aux recommandations du fabricant (Alpha Diagnostic International). Les résultats d'analyse ont été interprétés en fonction de la densité optique (DO). Chaque DO a été divisée par le seuil de positivité, ainsi un échantillon a été considéré positif lorsque le ratio obtenu étant supérieur à 1 avec un seuil de positivité de 0,492 .

\section{Analyse statistique}

Le traitement des données a été fait avec le logiciel $\mathrm{R}$ version 3.1.0. Un test de chi carré a été réalisé pour évaluer l'association entre les variables qualitatives âge, commune, sexe et infestation par les tiques. Lorsque les variables ne suivaient pas une distribution normale, des tests non paramétriques, tels que le test de Kruskall Wallis ont été réalisés pour les comparaisons multiples. Le seuil de signification a été fixé à $\mathrm{p}<0,05$. 


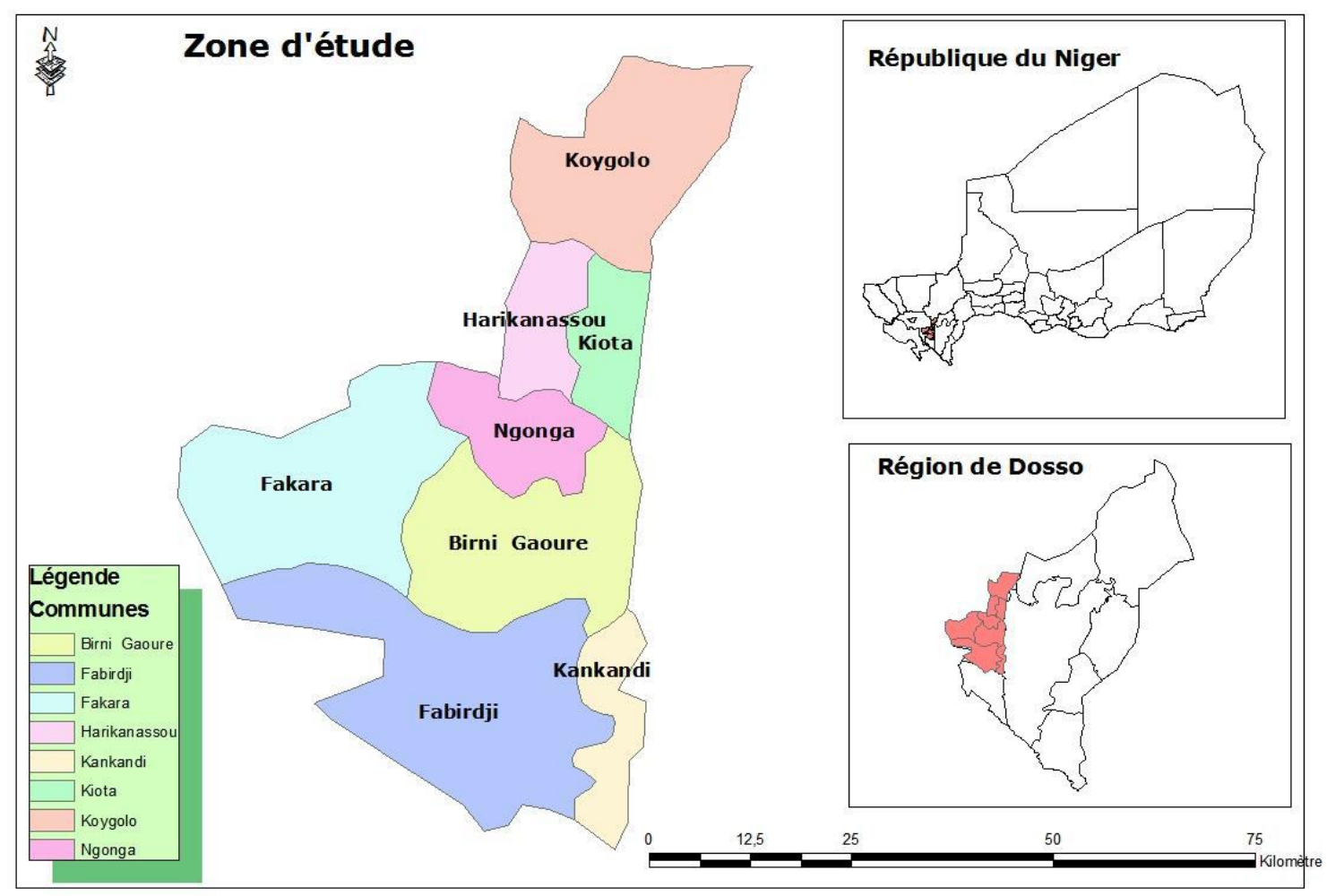

Figure 1 : Zone de l'étude.

\section{RÉSULTATS}

\section{Enquête de perception}

Soixante-douze éleveurs, tous des hommes dont l'âge variait de 15 à 60 ans, ont été interrogés dans les 8communes. Les résultats ont montré que $76,4 \%$ d'entre eux disaient avoir effectué une transhumance, en majorité vers la frontière du Mali, et 90,3\% affirmaient que d'autres éleveurs se déplaçaient en sens inverse vers leur région d'origine. Pour lutter contre les tiques, 20,8\% des éleveurs ont affirmé avoir eu recours à l'usage d'acaricides commerciaux comme l'ivermectine. Par contre, 51,4\% pratiquent le détiquage manuel, $10 \%$ font le système de brûlage et enfin 17,8\% n'envisagent aucune action. Pour les éleveurs interrogés les tiques sont globalement plus abondantes en saison sèche $(68,1 \%)$ que pluvieuse $(59,7 \%)$.

\section{Identification des tiques}

Au total, 1342 tiques ont été collectées, dont 81à Birni Gaouré, 229 à Fabirdji, 192 à
Kankandi, 282 à Harikanassou, 80 à Koygolo, 286 à Ngonga, 96 à Fakara et 96 Kiota.(Tableau 1). Elles appartenaient à trois genres, Hyalomma, Amblyomma et Rhipicephalus (Boophilus). Hyalomma était prédominante dans toutes les communes. Il y avait une différence significative entre l'infestation par les tiques et les communes, montrant ainsi que les tiques sont plus présentes dans certaines communes. Par exemple, le nombre le plus élevé de tiques collectées est de 286 à Harikanassou contre 80 à Birni Gaouré. Le nombre de tique du genre Hyalomma par commune est de 62 à Birni Gaouré, 208 à Fabirdji, 153 à Kankandi, 277 à Harikanassou, 80 à Koygolo, 271 à Ngonga, 84 à Fakara et 96 Kiota (Tableau 1).

\section{Séroprévalence}

Sur les 355 sérums de bovins analysés, 32 ont été positifs, soit une prévalence sérologique globale de $9,01 \pm 3 \%$. Cette prévalence a considérablement varié en 
fonction des communes puisqu'il n'y avait aucun sérum positif dans la moitié des communes, alors que la séropositivité était de 26\% pour Harikanassou (Tableau 2). Il y avait d'autre part une différence significative en fonction du sexe, mais celle observée en fonction de l'âge des animaux n'était pas significative (Tableau 3).

Tableau 1 : Répartition du nombre de tiques collectées selon les communes et le genre.

\begin{tabular}{lcccc}
\hline Communes & \multicolumn{3}{c}{ Genre } & \\
\hline & Hyalomma & Amblyomma & Boophilus & Total \\
\cline { 2 - 4 } Kiota & 96 & 0 & & 96 \\
Harikanassou & 277 & 0 & 0 & 282 \\
Ngonga & 271 & 15 & 5 & 286 \\
Koygolo & 80 & 0 & 0 & 80 \\
Birnin Gaouré & 62 & 19 & 0 & 81 \\
Kankandi & 153 & 18 & 0 & 192 \\
Fakara & 84 & 12 & 21 & 96 \\
Fabirdji & 208 & 12 & 0 & 229 \\
\hline Total & $\mathbf{1 2 3 1}$ & $\mathbf{7 6}$ & 9 & $\mathbf{1 3 4 2}$ \\
\hline
\end{tabular}

Tableau 2 : Séroprévalence de la Fièvre Hémorragique de Crimée Congo par Commune.

\begin{tabular}{lccccc}
\hline Communes & $\begin{array}{c}\text { Taille de } \\
\text { l'échantillon }\end{array}$ & $\begin{array}{c}\text { Nombre de } \\
\text { séropositifs }\end{array}$ & $\begin{array}{c}\text { Prévalence } \\
(\boldsymbol{\%})\end{array}$ & \multicolumn{2}{c}{ IC } \\
\cline { 2 - 7 } Kiota & 40 & 9 & 22,5 & 9,6 & 35,4 \\
Harikanassou & 45 & 12 & 26,66 & 13,7 & 39,6 \\
Ngonga & 45 & 4 & 8,88 & 0,6 & 17,2 \\
Birni Gaouré & 45 & 7 & 15,55 & 5 & 26,1 \\
Kankandi & 45 & 0 & 0 & 0,00 & 0,00 \\
Fabirdji & 45 & 0 & 0 & 0,00 & 0,00 \\
Fakara & 45 & 0 & 0 & 0,00 & 0,00 \\
Koygolo & 45 & 0 & 0 & 0,00 & 0,00 \\
\hline Total & 45 & 32 & 9,01 & 6 & 12 \\
\end{tabular}

IC : Intervalle de confiance.

Tableau 3 : Séroprévalence en fonction du sexe et de l'âge.

\begin{tabular}{lccccc}
\cline { 2 - 6 } & \multicolumn{2}{c}{ Sexe } & \multicolumn{3}{c}{ Âge } \\
\cline { 2 - 6 } & Femelles & Mâles & Jeunes & Adultes & Vieux \\
\cline { 2 - 6 } Nb. de sérum & 212 & 143 & 57 & 177 & 121 \\
Nb. de positif & 26 & 6 & 7 & 14 & 11 \\
Prévalence & $\mathbf{1 2 , 3 \%}$ & $\mathbf{4 , 1 9 \%}$ & $\mathbf{1 2 , 2 8 \%}$ & $\mathbf{7 , 9}$ & $\mathbf{9 , 0 9 \%}$
\end{tabular}

Jeunes (1-2) ans ; Adultes (3-8) ans ; vieux (9-15) ans. 


\section{DISCUSSION}

La présente étude a été conduite dans le département de Boboye (région de Dosso) qui est une zone humide à forte concentration de bétail. C'est une zone de parcours des transhumants où plus de la moitié des éleveurs pratique un détiquetage manuel pour lutter contre les tiques contrairement aux éleveurs en Côte d'Ivoire qui utilisent des acaricides (Touré et al., 2012).

Les résultats obtenus démontrent une prédominance de la tique vectrice de la FHCC à savoir Hyalomma spp au niveau des huit communes de la zone d'étude. En effet, Hyalomma spp est une tique qui vit dans les zones à biotope chaud et où les précipitations sont dans un intervalle comprenant les pluviométries annuelles enregistrées dans la zone de l'étude.

Le genre Amblyomma infestant les ruminants domestiques notamment les bovins dans la zone d'étude, est présent dans les 5 communes, particulièrement dans celles qui sont plus au Sud. C'est une tique dont l'activité est saisonnière en zone sahélo-soudanienne, où tombent moins de $1250 \mathrm{~mm}$ de pluies annuelles, et où l'année est divisée en une saison sèche et une saison de pluie. Il existe une seule génération par an, les adultes deviennent actifs dès les premières pluies et commencent à disparaître à la fin des pluies (Farougou et al., 2007). C'est qui expliquerait le faible nombre Amblyomma retrouvées dans la présente étude, puisque pratiquement tous les prélèvements ont été faits au cours du mois deseptembre, coïncidant avec la régression de la pluviométrie au Niger. Cela expliquerait le faible nombre de tiques du genre Amblyomma récoltées lors de cette étude, puisque pratiquement les prélèvements ont été faits au cours du mois septembre coïncidant avec la régression de la pluviométrie au Niger. Toutefois l'environnement de la zone d'étude est plus favorable à la prolifération de Hyalomma par à Amblyomma.

Le genre Boophilus a été identifié majoritairement dans deux communes situées à l'extrême sud (Fabirgi et Kankandi) plus humides. En effet le genre Boophilus prolifère plus dans des zones très humides (Adakal et al., 2013 ; Boka et al., 2014).

La prévalence sérologique globale observée est de 9\%, un résultat nettement inférieur à celui obtenu chez les bovins au Mali (66\%) et en Mauritanie (67\%) (Maiga et al, 2017; Sas et al 2017). Les résultats sérologiques ont varié également selon les communes. En effet, les bovins de Harikanassou et Kiota démontrent une séroprévalence plus élevée que les bovins des autres communes à $p<0,05$. Cette différence pourrait être due au fait que dans ces deux communes proches l'une de l'autre, les éleveurs partagent les mêmes pâturages, les tiques ont été assez abondantes, et surtout que celles-ci servent à la fois de vecteur mais aussi de réservoir pour le virus de la FHCC (Whitehouse, 2004). Par ailleurs, la disparité des prévalences selon des endroits peut être due à la densité des animaux dans la zone comme en témoignent les résultats obtenus par Maiga et al. (2017). En plus, la possible transmission horizontale et verticale du virus chez les tiques est un facteur contribuant au maintien durable de la maladie (Gargili et al., 2017). Étant donné que les anticorps anti-FHCC restent à vie chez les bovins, il se peut aussi que l'infection ait pu survenir des mois ou des années avant notre étude dans ces deux communes. Les facteurs climatiques comme la température, l'humidité et la pluviométrie ont une influence importante sur la distribution des tiques vectrices de la FHCC (Hoeket al., 2012). Les tiques telles que Hyalomma marginatum et Amblyomma variegatum sont connues pour être impliquées dans la distribution du virus de la fièvre hémorragique de Crimée-Congo (Zientara, 2013). Seule la recherche du génome viral pourra établir véritablement avec certitude une relation entre la présence de tiques vectrices de la CCHF et la séroprévalence.

La sérologie a été positive chez les jeunes bovins et aussi chez les adultes, mais la différence n'est pas significative. Ce fait, rend 
à lui seul en évidence la circulation du virus dans la zone d'étude. Par contre, les femelles ont été significativement plus infestées que les mâles. Dans le cas où le virus est transmis par les tiques collectées, ce résultat peut être expliqué par le fait que l'infestation a été plus importante chez les femelles au moment de l'étude mais aussi, dans ces élevages les femelles sont gardées plus longtemps, donc plus de chance de rencontrer le virus. Dans le cas contraire, la transmission du virus pourrait intervenir par d'autres types de vecteurs.

\section{Conclusion}

La présente étude avait comme objectif de contribuer à une meilleure connaissance de l'épidémiologie de la Fièvre Hémorragique de Crimée-Congochez les bovins dans le département de Boboye. Les résultats obtenus démontrent une faible prévalence sérologique de la FHCC chez les bovins, mais témoignent de la circulation du virus chez cette espèce animale. Cela signifie un risque élevé d'exposition au virus, en particulier pour les humains notamment lors de l'abattage ou de la consommation de lait non pasteurisé. Aucune déclaration de la maladie n'a été faite chez les humains au Niger. Les personnes à risque doivent être informées de la maladie et des mesures de surveillances appropriées doivent être mises en œuvre (dépistage de la maladie) surtout qu'en janvier 2020, qu'un pays voisin du Niger (Mali) a déclaré une épidémie sur son territoire.

\section{CONFLIT D'INTERETS}

Les auteurs affirment que dans l'élaboration de la présente étude, il n'existe aucun conflit d'intérêts.

\section{CONTRIBUTIONS DES AUTEURS}

AM est une étudiante qui a travaillé dans le cadre de son mémoire de Master sur le thème. AII a suivi le travail du terrain et du laboratoire et également aussi contribué à la rédaction du présent article. AA a contribué à la correction du manuscrit. HA a contribué à l'élaboration du protocole et à la correction du manuscrit.

\section{REMERCIEMENTS}

Les auteurs tiennent à exprimer leur gratitude au Programme de Productivité Agricole en Afrique de l'Ouest (PPAAO/Niger) pour le financement de cette recherche.

\section{REFERENCES}

Adakal H, Biguezoton A, Zoungrana S, Courtin F, De Clercq EM, Madder M. 2013. Alarming spread of the Asian cattle tick Rhipicephalus microplus in West Africa-another three countries are affected: Burkina Faso, Mali and Togo. Exp. Appl. Acarol., 61(3): 383-386. DOI: 10.1007/s10493-013-9706-6.

Boka OM, Madder M, Achi YL, Kaboret YY, Berkvens D. 2014. Modélisation du Remplacement de la Tique Rhipicephalus (Boophilus) Decloratus (Boophilus) par Microplus, une Tique Emergente en Côte d'Ivoire. European Scientific Journal, 10(30): 120-132.

Farougou S, Tassou AW, Tchabode DM, Kpodekon M, Boko C, Youssao AKI. 2007. Tiques et hémoparasites du bétail dans le nord Bénin. Rev. Elev. Med. Vet. Pays Trop., 158 : 463-467.

Gargili A, Estrada-Peña A, Spengler JR, Lukashev A, Nuttall PA, Bente DA. 2017. The role of ticks in the maintenance and transmission of Crimean-Congo hemorrhagic fever virus: a review of published field and laboratory studies. Antiviral Res., 144:93-119. DOI: 10.1016/j.antiviral.2017.05.010.

Hoek M, Fischer E, Hengel RVD, Backer J, Koeijer AD. 2012. Workshop on a Risk Assessment of Crimean-Congo Haemorrhagic Fever in Western Europe, Lelystad, Netherlands, 23-24 May 2012.

Institut National de la Statistique (INS). 2014. Le Niger en chiffres. Rapport de la 
Direction Générale de l'Institut National de la Statistique, $84 \mathrm{p}$.

Maiga O, Andrada Sas M, Rosenke B, Kamissoko B, Mertens M, Sogoba N, Traoré, A, Sangaré M, Niang M, Schwan TG, Maiga HM, Traore SF, Feldmann H, Safronetz D. 2017. Serosurvey of Crimean-Congo Hemorrhagic Fever Virus in Cattle, Mali, West Africa.Am. J. Trop. Med. Hyg., 96(6): 1341-1345. DOI: $10.4269 /$ ajtmh.16-0818.

Organisation Mondiale de la Santé (OMS), 2013. Fièvre Hémorragique de CriméeCongo. Aide-mémoire, $\quad \mathrm{N}^{\circ} 208$., http://www.who.int/mediacentre/factshee ts/fs208/fr/ (consulté le 05/05/2019).

Papa A, Tsergouli K, Tsioka K, Mirazimi A. 2017. Crimean-Congo Hemorrhagic Fever: Tick-Host-Virus Interactions. Front. Cell. Infect. Microbiol., 7: 213. DOI: $\quad 10.3389 /$ fcimb.2017.00213. eCollection.

Saluzzo JF, Camicas JL, Chartier C, Martinez D, Digoutte JP. 1986. Le virus de la Fièvre Hémorragique de Crimée-Congo (CCFH) en Mauritanie. Cah. - ORSTOM, Entomol. Méd. Parasitol., 24(2): 129137.
Saluzzo JF, Vidal P, Gonzalez JP. 2004. Les virus émergents. IRD, Paris, France, 190 p.

Sas MA, Mertens M, Isselmou E, Reimer N, Mamy BOEL, Doumbia B, Groschup MH. 2017. Crimean-Congo hemorrhagic fever virus specific antibody detection in cattle in Mauritania. Vector Borne Zoonotic Dis., 17(8): 582-587. DOI: 10.1089/vbz.2016.2084.

Touré A, Komoin-Oka C, Sylla I. 2012. Cattle ticks population and prevalence of Babesia sppamongst it vector: Rhipicephalus (Boophilus) microplusin a zone of Ivory Coast. Int. J. Biol. Chem. Sci., 6(4): 1514-1581. DOI: http://dx.doi.org/10.4314/ijbcs.v6i4.17.

Walker AR, Bouattour A, Camicas JL, EstradaPeña A, Horak IG, Latif AA, Pegram RG,

Whitehouse CA. 2004. Crimean-Congo hemorrhagic fever. Antiviral Res., 64(3): 145-160.

DOI: 10.1016/j.antiviral.2004.08.001.

Zientara S. 2013. La fièvre hémorragique de Crimée-Congo est en recrudescence en Europe orientale. Bulletin Epidémiologique., 33: 13. 